\title{
Prediction of Patient-Specific Tissue Engineering Scaffolds for Optimal Design
}

\author{
Yong L. Chuan, Md. E. Hoque, and Ian Pashby
}

\begin{abstract}
The fabrication of patient-specific tissue engineering scaffold is highly appreciated that requires prior estimation of porous and mechanical characteristics. Architectural controllability and reproducibility are also essential aspects in the development of 3D functional scaffolds. This work presents a computational approach to determine porous and mechanical characteristics of $3 \mathrm{D}$ scaffolds. The computational modeling could be a powerful tool to assist designing 3D scaffold with optimum characteristics as required for a particular patient in need. The 3D scaffolds were successfully modeled investigating the influences of design parameters on the porous and mechanical properties via finite element analysis (FEA) and ANSYS application software. It was revealed by ANSYS that the increase in porosity decreased the mechanical properties and increased the damping factor. The Scaffold porosities were obtained in the range of $47 \%$ to $95 \%$ with varying pore shape and size by modulating lay-down pattern, filament diameter and filament distance.
\end{abstract}

Index Terms-Tissue engineering, scaffold, rapid prototyping, finite element analysis.

\section{INTRODUCTION}

Tissue engineering (TE) and guided tissue repair are very rapidly developing new areas of science. TE is evolving discipline that seeks to repair, replace, or regenerate specific tissues or organs by translating fundamental knowledge in physics, chemistry, and biology into practical and effective materials, devices, systems, and clinical strategies [1], [2]. The principles of TE is that tissues can be isolated from a patient, expanded in tissue culture and seeded into a scaffold prepared from a specific building material to form a scaffold guided three-dimensional (3D) tissue. The construct can then be grafted into the same patient to function as a replacement tissue [3].

Many scaffolds used as medical implants and for TE purposes are fabricated by conventional methods (i.e., expanded grafts, textile weaves and braids, porous films, and sponges). These methods are limited in that they typically generate scaffolds with simple macro-architectures and homogeneous microstructures [4]. Critical variables in scaffold design and function include the bulk material or materials from which it is made, the $3 \mathrm{D}$ architecture, the surface chemistry, the mechanical properties, the initial environment in the area of the scaffold, and the late scaffold environment, which is often determined by degradation

Manuscript received April 30, 2013; revised July 22, 2013.

Yong L. Chuan is with the INTI International University (e-mail: yonglengchuan@gmail.com).

MD. E. Hoque and Ian Pashby are with the University of Nottingham Malaysia Campus. characteristics. 3D porous scaffolds promote new tissue formation by providing a surface and void volume that promotes the attachment, migration, proliferation, and desired differentiation of connective tissue progenitors throughout the region where new tissue is needed [5], [6].

Jaecques et al. (2004) [7] have performed a stress-strain aalysis of complete scaffolds via FEA to investigate the state of stress and strain within the scaffolds and its interaction with the surrounding tissues [8], [9]. Such analysis can be used to vary several geometrical or material parameters at the same time and to choose the most suitable ones for the replacement of natural tissues [7]. Simulations of perfusion bioreactors have been investigated for 3D scaffold performances [10], [11], [12]. Lacroix, D., and Prendergast, P.J. (2002) [13], [14] studied tissue differentiation and bone regeneration as functions of the porosity, Young's modulus, dissolution rate, load condition, and recommended these as the possible optimal scaffold parameters that can be controlled by RP fabrication.

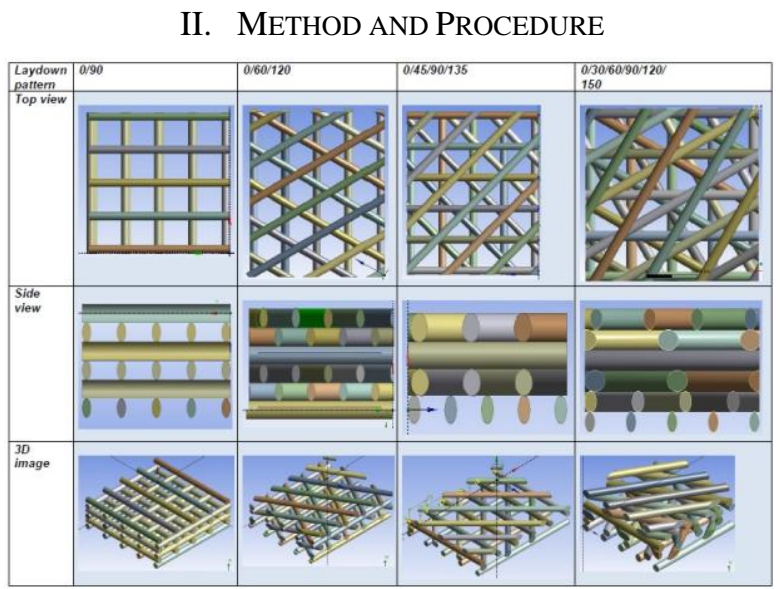

Fig. 1. TE scaffolds with different architectures

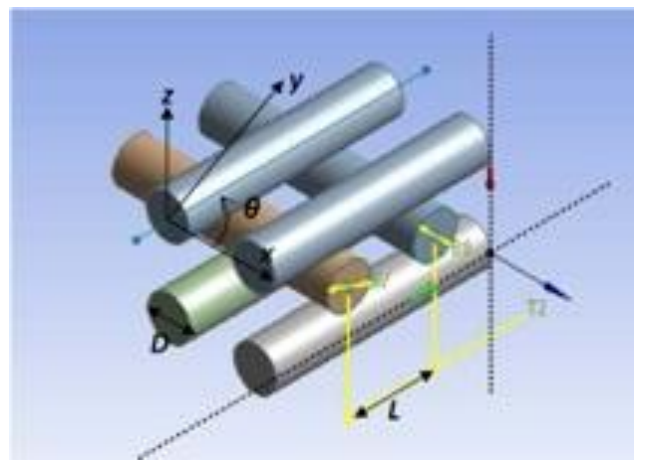

Fig. 2. Schematic diagram for porosity calculation

Cubic porous scaffolds $(25 \mathrm{~mm} \times 25 \mathrm{~mm} \times 25 \mathrm{~mm})$ were designed with various architectures by varying design 
parameters by ANSYS 12 software. Four different architectural designs were generated for mechanical simulation. Firstly, all possible parameters were listed down without any concern of mechanical properties and fabrication possibility. Various scaffold architectures can be imprinted by applying various lay-down patterns (0-90, 0/60/120, $0 / 45 / 90 / 135$, and 0/30/60/90/120/150) as shown in Fig. 1 below using appropriate positioning of the robotic control system. The lay-down patterns of $0-90,0 / 60 / 120$, 0/45/90/135, and 0/30/60/90/120/150 are also called 2-angle, 3 -angle, 4-angle and 6-angle patterns, respectively. Fig. 1 shows all possible design which evaluate in this part. Secondly, all designs porosity and tensile properties were mathematically evaluated by MATLAB software. Fig. 2 summarizes a flow chart of main characterization and scaffold design filter for the whole computerization analysis. The scaffolds with feasible parameters were analyzed for compression properties via FEA. The obtained mechanical properties for different parameters served as guideline for the optimization process on how the material properties and process parameters can be combined to obtain desired scaffold properties. The scaffolds' biomechanical properties can be justified through in hand experimental evaluation.

The porosity of different scaffolds was estimated using MATLAB R2007a (The Mathworks Inc.) software. The porosity was calculated as the percentage of void voxels relative to the total number of voxels within the scaffold model. The porosity $(\mathrm{P})$ of the scaffolds was calculated by:

$$
\mathrm{P}=1-(\pi \mathrm{D} / 16 \mathrm{G})(1+1 / \sin \alpha)
$$

where $L$ is the centre to centre filament gap, $\alpha$ is the filament orientation angle and D is the filament diameter (Fig. 2).

\section{RESULT AND DISCUSSION}

The porosity of various scaffolds was estimated by means of MATLAB software. Computational simulation was carried out for the various design variables filament gap (L), filament diameter (D) and lay-down angle. Then, for the optimal design of the porous architecture, the evaluation function of filament gap and filament diameter obtained by computational simulation. The porosity can be easily calculated from the design variable $\mathrm{L}$ and $\mathrm{D}$ for the designed architecture. The porosity (\%) values were found to be in the range of $47-87 \%$ depending on the scaffold design/architecture. The porosity from top to bottom throughout the scaffold height was assumed to be the same as expected from precise fabrication method involving exact placement of the filaments during layer-by-layer deposition by controlled RP system. The porosity found to be directly controlled by the design parameters as the porosity was increased with the increase of filament gap and lay down angle. The porosity plays significant role in the regeneration of tissue through controlling scaffold permeability and mechanical property, and cell growth [15]. The higher the porosity, the more space is available in the scaffold for the formation of new tissue. The porosity should preferably as high as possible. However, the filament gap that induces the porosity should be corresponding to the filament diameter which means that the ratio of filament gap to filament diameter should be within a practical range. In reality, if the value of this ratio is above 3 the integrity of the fabrication process is hampered and stability of the scaffold structure is lost [16]. It is because of slacking of the filaments during polymer deposition, which ultimately interrupts the pore interconnectivity. It is in agreement that a highly interconnected channel network in the scaffold was essential for flow transport of nutrients and wastes in vivo [17], [18].

The increase of filament distance increased porosity and accordingly decreased the yield strength of the scaffold. In this study, a linear correlation was used to fit the data points, whereas the stiffness of an open porous material is usually represented as a function of the square of the porosity. Similar trends were obtained by [13], [14], [19], [20]. The mechanical properties curves for samples with the same material are very similar, allowing the comparisons of the mechanical response of different geometry (Fig. 3).

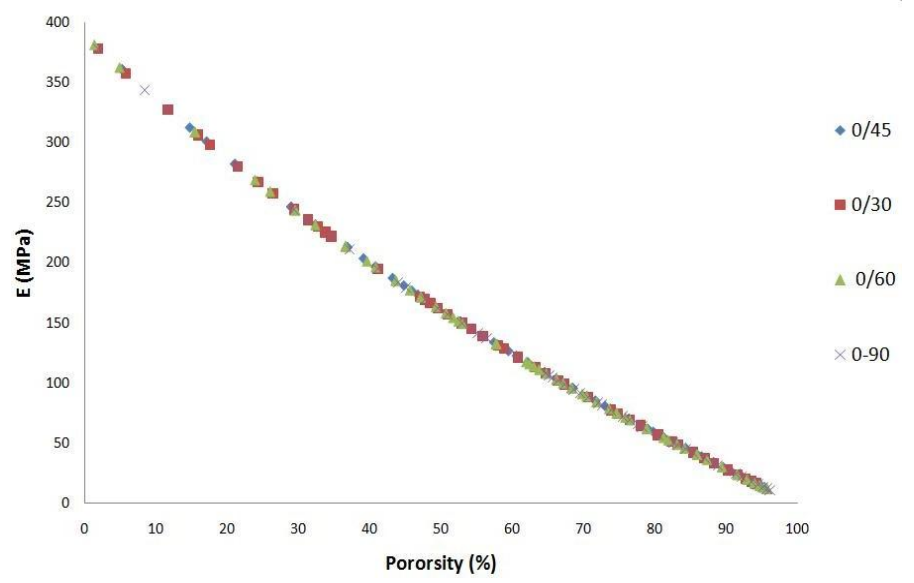

Fig. 3. Relationship between effective Young's modulus and scaffold porosity for a various scaffolds architectures. The effective Young's modulus was defined as the axial stress (reaction force divided by area) over the axial strain $(0.5 \%)$

\section{CONCLUSION}

Modelling, simulation and optimization of 3D scaffold done so far here in terms of porosity and mechanical characterizations were well defined. Computational models were developed for various 3D scaffold designs by varying design parameters namely, lay-down pattern, filament diameter and filament distance which control the porous characteristics and consequently, mechanical properties. The porous characteristics of designed 3D scaffolds were evaluated by MATLAB, while the finite element analysis (FEA) was performed with ANSYS to determine mechanical properties. Based on the mathematical and finite element analysis it could be concluded that the design parameters (filament diameter, filament distance/gap and lay-down angle) have direct influences on the scaffold porosity and mechanical properties. It was revealed by ANSYS that the increase of porosity decreased the mechanical properties and increased the damping factor. Compressive test results revealed that the scaffold's mechanical properties were directly controlled by the filament diameter and distance. An anisotropic behavior of the scaffold was also observed which indicated that the mechanical properties were strongly 
dependant on the scaffold's filament lay-down orientation. This computational model can be a useful tool to predict scaffold architectures and properties and ultimately to fabricate scaffold by means of our in house built desktop robot-based rapid prototyping (DRBRP) system. A scaffold library can be developed upon successful completion of detailed study on the relationships among the scaffold materials, architectures and properties. The scaffold library can be a powerful database which would guide the tissue engineers to manufacture scaffold with required characteristics for patient-specific tissue engineering applications. This systematic computational approach could assist in optimizing 3D scaffold development for a patient-specific application by minimizing the need of physical experiments. These computational data would be utilized to develop physical 3D scaffolds using RP technology in combination with synthetic biopolymers.

\section{REFERENCES}

[1] G. F. Muschler, C. Nakamoto, and L. G. Griffith, "Engineering principles of clinical cell-based tissue engineering," J. Bone Joint Surg Am, vol. 86, pp. 1541-1558, 2004.

[2] G. Tripathi and B. Basu, "A porous hydroxyapatite scaffold for bone tissue engineering: Physico-mechanical and biological evaluations," Ceramics International, vol. 38, no. 1, pp. 341-349, 2012.

[3] A. Lucke, J. Tebmar, E. Schnell, G. Schmeer, and A. Gopferich, "Biodegradable poly (D, L-lactic acid)-poly (ethylene glycol)-monomethyl ether diblock copolymers: structures and surface properties relevant to their use as biomaterials," Biomaterials, vol. 21 , pp. 2361-2370, 2000.

[4] J. Zeltinger, "Effect of pore size and void fraction on cellular adhension, poliferation, and matrix deposition," Tissue Engineering, vol. 7, pp. 5, 2001.

[5] S. I. Roohani-Esfahani, Z. F. Lu, J. J. Li, R. Ellis-Behnke, D. L. Kaplan, and H. Zreiqat, "Effect of self-assembled nanofibrous silk/polycaprolactone layer on the osteoconductivity and mechanical properties of biphasic calcium phosphate scaffolds," Acta Biomaterialia, vol. 8, no. 1, pp. 302-312, 2012.

[6] I. Springer, B. Fleiner, S. Jepsen, and Y. Acil, "Culture of cells gained from temporomandibular joint cartilage on non-absorbable scaffolds," Biomaterials, vol. 22, pp. 2569-77, 2001.

[7] S. V. N. Jaecques, H. V. Oosterwyck, L. Muraru, T. Van Cleynenbreugel, E. D. Smet, and M. Wevers, "Individualised, micro CT-based finite element modelling as a tool for biomechanical analysis related to tissue engineering of bone," Biomaterials, vol. 25, pp. 1683-1696, 2004.

[8] A. K. Burkoth and K. S. Anseth, "A review of photocrosslinked polyanhydrides: in situ forming degradable networks," Biomaterials, vol. 21, pp. 2395-2404, 2000

[9] M. Lebourg, R. S. Serra, J. M. Estelles, F. H. Sanchez, J. L. G. Ribelles, and J. S. Anton, "Biodegradable polycaprolactone scaffold with controlled porosity obtained by modified particle-leaching technique,' Journal of Materials Science, vol. 19, pp. 2047-2053, 2008.

[10] M. E. Hoque, Y. L. Chuan, and I. Pashby, "Extrusion based rapid prototyping technique - An advanced platform for tissue engineering scaffold fabrication," Biopolymers, vol. 97, pp. 83, 2012.

[11] T. V. Cleynenbreugel, J. Schrooten, V. Oosterwyck, and J. Vander Sloten, "Micro-CT-based screening of biomechanical and structural properties of bone tissue engineering scaffolds," Medical and Biological Engineering and Computing, vol. 44, pp. 517-525, 2006.

[12] C. Margherita, B. Federica, T. Manuela, and D. Gabriele, "Modeling evaluation of the fluid-dynamic microenvironment in tissue-engineered constructs: A micro-CT based model," Biotechnology and Bioengineering, vol. 93, pp. 500-510, 2006.

[13] D. Lacroix and P. J. Prendergast, "A mechano-regulation model for tissue differentiation during fracture healing: analysis of gap size and loading," Journal of Biomechanics, vol. 35, pp. 1163-1171, 2002.

[14] D. Lacroix, A. Chateau, M. Ginebra, and J. Planell, "Micro-finite element models of bone tissue-engineering scaffolds," Biomaterials, vol. 27, pp. 5326-5334, 2006.
[15] C. Sandino, J. A. Planell, and D. Lacroix, "A finite element study of mechanical stimuli in scaffolds for bone tissue engineering," Journal of Biomechanics, vol. 41, pp. 1005-1014, 2008.

[16] L. Shor, "Fabrication of 3D PCL/HA Tissue Scaffolds and osteoblast-scaffold interactions in vitro," Biomaterials, vol. 28, pp. 5291-5297, 2007.

[17] M. J. Silva, W. C. Hayes, and L. J. Gibson, "The effects of non-periodic microstructure on the elastic properties of two-dimensional cellular solids," International Journal of Mechanical Sciences, vol. 37, pp. 1161-1177, 1995.

[18] B. Cohen, "Navigating through tissue expansion terminology," $J$. Dermatol Surg Oncol, vol. 19, pp. 614-615, 1993.

[19] A. Boger, N. Bhadra, and K. J. Gustafson, "Voiding by combined high frequency electrical pudendal nerve block and sacral root stimulation," Neurourol Urodyn, vol. 27, pp. 435-439, 2008.

[20] L. J. Gibson, K. Anthony, and Z. Carl, "Properties and Applications of Metallic Foams," in Comprehensive Composite Materials. Oxford: Pergamon, 2000, pp. 821-842.

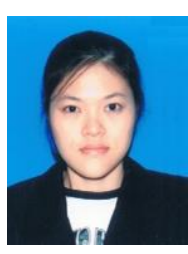

Yong L. Chuan gradutated from National University of Malaysia for BSc (Hons) Materials Science (2006) and MSc Materials Science (2010). She has received hers $\mathrm{PhD}$ in Mechanical Engineeing from University of Nottingham, Malaysia Campus, in year 2013.

Dr. Yong Leng Chuan is a lecturer in the School of Engineering. She has been intensively involved in numerous research projects that are collaborated locally and internationally. Hers research interests include the areas of rapid prototyping technology, biomaterials, polymer, tissue engineering, and nanomaterials.

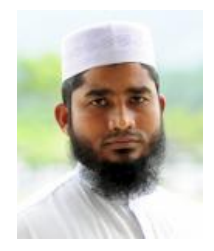

Md E. Hoque received his Ph.D. in Bioengineering from NUS, Singapore in 2007.

$\mathrm{He}$ is an Associate Professor in the Department of Mechanical, Materials \& Manufacturing Engineering and served as Head for the Bioengineering Research Group at the University of Nottingham Malaysia Campus. He has been intensively involved in numerous research projects that are collaborated locally and internationally. So far, he has authored TWO books, co-authored THREE book chapters and edited TWO books. He has also published more than 65 technical papers in referred journals and international conference proceedings. His research interests include the areas of Rapid Prototyping Technology, Biomaterials, Biofuel, Tissue Engineering, Stem Cells, Nanomaterials and Composite Materials.

Dr. Muhammad Enamul Hoque is an editorial manager for the Journal of Applied Mechanical Engineering and an invited lead guest editor of the International Journal of Materials and Mechanical Engineering, and serves as a technical reviewer for about 10 international journals. He has been invited to deliver speeches in a number of international conferences. He has also organized several scientific conferences assuming various roles in the organizing committees. He is a member of several professional scientific societies including Tissue Engineering and Regenerative Medicine International Society (TERMIS), and Tissue Engineering Society Malaysia (TESMA).

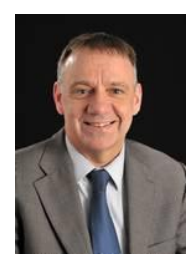

Ian Pashby graduated from the University of Sheffield, metallurgist by education and was awarded his $\mathrm{PhD}$ from University of Warwick in 1992.

He took up the post of Pro-Vice-Chancellor of University of Hull, United Kingdom on 1 January 2013. He has been worked in alloy and material processing research for Rolls-Royce aero engines. He joined the Warwick Manufacturing Group at the University of Warwick as a lecturer in 1992. Moving to the University of Nottingham in 2000 as Professor of Manufacturing Processes, Professor Pashby undertook roles as Head of the Advanced Manufacturing Technology research group and Deputy Head of the School of Mechanical, Materials and Manufacturing Engineering. In 2007 he was seconded to Nottingham's Malaysia Campus, initially as Deputy Vice-President but from 2008 as Provost and Chief Executive Officer.

Professor Pashby's research interests cover the processing of materials, specifically in the context of aerospace manufacturing, and particularly using nonconventional means such as electro discharge machining, waterjets and lasers. 